\title{
ON THE TOPOLOGICAL COMPLETION
}

\author{
HOWARD CURZER AND ANTHONY W. HAGER ${ }^{1}$
}

\begin{abstract}
Let $X$ be a Tychonoff space. As is well known, the points of the Stone-Čech compactification $\beta X$ "are" the zero-set ultrafilters of $X$, and the points of the Hewitt real-compactification $v X$ are the zero-set ultrafilters which are closed under countable intersection. It is shown here that a zeroset ultrafilter is a point of the Dieudonne topological completion $\delta X$ iff the family of complementary cozero sets is $\sigma$-discretely, or locally finitely, additive. From this follows a characterization of those dense embeddings $X \subset Y$ such that each continuous metric space-valued function on $X$ extends over $Y$, and a somewhat novel proof of the Katětov-Shirota Theorem.
\end{abstract}

All spaces shall be Tychonoff.

It is most convenient to view the class of topologically complete spaces as the class $\Re(\Re)$ of closed subspaces of products from the class $\mathscr{R}$ of metrizable spaces, that is, as the epireflective hull of $\Re$. (Dieudonné showed that a Tychonoff space $X$ has a compatible complete uniformity iff $X$ admits an embedding with closed range into a product of metrizable spaces [D].) The topological completion $\delta X$ of a Tychonoff space $X$ is the epireflection of $X$ into $\Re(\Re)$, that is, $\delta X$ is the essentially unique topologically complete space containing $X$ densely such that each continuous map $f: X \rightarrow Z(Z \in \Re(\Re))$, admits a continuous extension $\delta f: \delta X \rightarrow Z$. This universal mapping property is implied by the weaker one for maps into spaces in $\mathscr{N}$, by the standard technique used to show for $\beta X$ that the universal mapping property for maps to $[0,1]$ implies the property for maps to compact spaces. See, e.g., [W]. We shall use this fact below.

$\delta X$ may be constructed as the closure of a suitable homeomorph of $X$ in a large product of metrizable spaces, similar to the common construction of $\beta X$ (e.g., [W]). The following is a more useful construction for our purpose. It depends on knowledge of $\beta X$.

1. Lemma. $\delta X=\bigcap\left\{(\beta f)^{-1}(M) \mid f: X \rightarrow M\right.$ continuous, $\left.M \in \mathfrak{T}\right\}$. (Here $\beta f: \beta X \rightarrow \beta M$ is the extension over the Stone-Čech compactifications.)

We sketch a proof of 1 . Let $Y=\cap_{M}\left\{(\beta f)^{-1}(M)\right\}$. Clearly, a continuous map $f: X \rightarrow M$ has the extension $\beta f \mid Y: Y \rightarrow M$, so it suffices to show that $Y \in \mathcal{R}(\Re)$. Since $\mathcal{R}(\Re)$ is productive and closed-hereditary, it is closed under intersection (seen by realizing an intersection as a diagonal in a product), so it

Received by the editors March 3, 1975.

AMS (MOS) subject classifications (1970). Primary 54B99, 54C45, 54D20, 54D35, 54E14.

Key words and phrases. Topological completion, zero-set ultrafilter, $\sigma$-discrete, locally finite, extension of functions, Katětov-Shirota Theorem.

${ }^{1}$ This author is pleased to thank the Academies of Science of Czechoslovakia and the United States for support. 
suffices that each $(\beta f)^{-1}(M) \in \mathscr{R}(\mathscr{R})$. Now $\mathscr{R}(\mathscr{R})$ is closed-hereditary, and $A \times K \in \Re(\Re)$ if $A \in \mathcal{R}(\Re)$ and $K$ is compact-since $K \in \mathscr{R}(\Re)$; this implies that $\Re(\Re)$ is closed under perfect pre-images [HS]. (A continuous map $g: A \rightarrow B$ is called perfect if $\beta g(\beta A-A) \subset \beta B-B$.) But clearly, each $\beta f \mid(\beta f)^{-1}(M)$ is perfect.

(Something close to 1 appears in [F], from which we have borrowed the proof. Also see $[\mathbf{H}]$.)

It might be noted that one can view the class of realcompact spaces as the epireflective hull $\Re(\mathrm{R})$ of the real line $R$ (i.e., closed subspaces of powers of $R$ ), and then construct the epireflection $v X$-the Hewitt realcompactification-just as above:

$$
v X=\cap\left\{(\beta f)^{-1}(R) \mid f \in C(X)\right\},
$$

$C(X)$ being the ring of real-valued continuous functions.

In any event, $X \subset \delta X \subset v X \subset \beta X$.

Recall (say from [GJ]) that the points of $\beta X$ and the $z$-ultrafilters on $X$ (i.e., ultrafilters in the family of zero-sets of functions in $C(X)$ ) are associated oneto-one by $p \leftrightarrow \mathscr{F}_{p}=\{Z \mid Z$ is a zero-set and $p \in \bar{Z}\}$ (the closure in $\beta X$ ); that $p \in v X$ iff $\mathscr{F}_{p}$ is closed under countable intersection; that for $p \in v X$ and $Z$ a zero-set, $Z \in \mathscr{F}_{p}$ iff whenever $Z=Z f,(f \in C(X))$, then $\beta f(p)=0$.

If $\mathscr{F}$ is a $Z$-ultrafilter on $X$, let $\operatorname{co} \mathscr{F}=\{X-Z \mid Z \in \mathscr{F}\}$. To say that $\mathscr{F}$ is closed under countable intersection is to say that co $\mathscr{F}$ is closed under countable union, or, as we shall say, $\sigma$-additive. We introduce the stronger addition property, characterizing the points of $\delta X$.

A family $\mathscr{D}$ of subsets of $X$ will be called discrete if there is a continuous pseudometric $d$ on $X$ and $\varepsilon>0$ such that if $A, B \in \mathscr{D}$ with $A \neq B$, then $d(A, B) \geqslant \varepsilon$. A family is $\sigma$-discrete if it is the union of countably many discrete subfamilies.

Finally, a family $\mathcal{G}$ of subsets of $X$ is discretely (or $\sigma$-discretely, or locally finitely) additive if $\cup \mathscr{D} \in \mathcal{G}$ whenever $\mathscr{D} \subset \mathcal{G}$ and $\mathscr{D}$ is discrete (or $\sigma$-discrete, or locally finite).

2. THEOREM. Let $p \in \beta X$. The following are equivalent.

(a) $p \in \delta X$

(b) co $\mathscr{F}_{p}$ is $\sigma$-discretely additive;

(c) co $\mathscr{F}_{p}$ is locally finitely additive.

The proof will use the association between continuous pseudometrics $d$ on $X$ and continuous functions $f$ from $X$ to metric spaces $M$; given $d,\langle M, \rho\rangle$ is the "metric identification" of $\langle X, d\rangle$ and $f$ is the natural projection; given $f: X \rightarrow\langle M, \rho\rangle$, set $d(x, y)=\rho(f(x), f(y))$. It follows that each $d$ possesses a continuous pseudometric expression $\delta d$ over $\delta X$; since $d(A, B) \geqslant \varepsilon$ implies $\delta d(A, B) \geqslant \varepsilon$, a discrete (or $\sigma$-discrete) family in $X$ is discrete (or $\sigma$-discrete) in $\delta X$. And, if $g: X \rightarrow Y$ is continuous, and $\mathscr{D}$ is discrete (or $\sigma$-discrete) in $Y$, then $g^{-1}(\mathscr{D})$ is discrete (or $\sigma$-discrete) in $X$.

Note this also: if $\mathscr{D}$ is a discrete family of subsets of $X$, then each point of $X$ has a neighborhood meeting at most one member of $\mathscr{Q}$. (If $d(D, E) \geqslant \varepsilon$ for $D \neq E$ in $\mathscr{D}$, then $\{x \mid d(x, p)<\varepsilon / 2\}$ is such a neighborhood of $p$.) Thus, if $\mathscr{D}$ consists of cozero sets, say $\mathscr{D}=\left\{\operatorname{coz} f_{D} \mid D \in \mathscr{D}\right\}$, then $f=\Sigma\left\{f_{D} \mid D \in \mathscr{D}\right\}$ $\in C(X)$, and $\operatorname{coz} f=\cup \mathscr{D}$. 
Proof of 2. (a) implies (b): Let $p \in \delta X$. Since $\delta X \subset v X$, co $\mathscr{F}_{p}$ is $\sigma$-additive, so we must show that co $\mathscr{F}_{p}$ is discretely additive. Let $\mathscr{D} \subset \operatorname{co} \mathscr{F}_{p}$, with $\mathscr{D}$ discrete. For $D \in \mathcal{D}$, choose $f_{D} \in C(X)$ with $D=\operatorname{coz} f_{D}$; thus $Z f_{D} \in \mathscr{F}_{p}$ and $\beta f_{D}(p)=0$. Set $f=\sum\left\{f_{D} \mid D \in \mathscr{D}\right\}$; as noted above, $f \in C(X)$ and coz $f$ $=\cup \mathscr{D}$. We show that $\beta f(p)=0$, i.e., that $\operatorname{coz} f \in \operatorname{co} \mathscr{F}_{p}$.

As noted above, $\left\{\operatorname{coz} \delta f_{D} \mid D \in \mathscr{D}\right\}$ is discrete in $\delta X$, so $\sum\left\{\delta f_{D} \mid D \in \mathscr{D}\right\}$ is well defined and continuous. By uniqueness of extension, $\Sigma_{D}\left\{\delta f_{D}\right\}=\delta f$. Thus, $\beta f(p)=\delta f(p)=\Sigma_{D}\left(\delta f_{D}\right)(p)$. This last is 0 , since for each $D, \delta f_{D}(p)$ $=\beta f_{D}(p)=0$.

(We have used the fact that for $p \in v X$, and $Z$ a zero-set, in order that $Z \in \mathscr{F}_{p}$, it is enough to find $f$ with $Z f=Z$ and $\beta f(p)=0$.)

(a) implies (c): It is possible to give an argument similar to the above, but the following is interesting.

Let $p \in \delta X$, and let $\mathscr{D}=\left\{\operatorname{coz} f_{D} \mid D \in \mathscr{D}\right\}$ be a locally finite subfamily of $\operatorname{co} \mathscr{F}_{p}$. As is well known and easily verified, $d(x, y) \equiv \Sigma_{D}\left|f_{D}(x)-f_{D}(y)\right|$ defines a continuous pseudometric on $X$; and $d$ has a continuous pseudometric extension $\delta d$ over $\delta X$. Let $Z_{n}=X \cap\{x \in \delta X \mid \delta d(x, p) \leqslant 1 / n\}$. Any set closed in the $\delta d$-topology is a zero-set of $\delta X$, and so $Z_{n}$ is a zero-set of $\delta X$. Clearly, $p \in \bar{Z}_{n}$, so that $Z_{n} \in \mathscr{F}_{p}$. Since $p \in v X, Z \equiv \cap_{n} Z_{n} \in \mathscr{F}_{p}$. Evidently, if $q \in Z$, then $\delta d(q, p)=0$. Finally, $Z \subset \cap_{D} Z f_{D}$ (so $\cap_{D} Z f_{D} \in \mathscr{F}_{p}$ ): for if $q \notin Z f_{D},\left|f_{D}(q)\right|>0$; since $d(x, y) \geqslant\left|f_{D}(x)-f_{D}(y)\right|$ for each $x, y$, it follows that

$$
\delta d(q, p) \geqslant\left|\delta f_{D}(q)-\delta f_{D}(p)\right|=\left|\delta f_{D}(q)\right|>0 .
$$

(c) or (b) implies (a): Essentially the same proof works in either case. Suppose that $p \notin \delta X$. By 1 , choose continuous $f: X \rightarrow M, M$ metrizable, with $\beta f(p) \notin M$. So $f$ fails to extend continuously to $p$ with values in $M$. Thus, fixing a metric $\rho$ on $M$, the oscillation of $f$ at $p$ is nonzero, say $\geqslant \varepsilon$. By A. H. Stone's Theorem [St] (or see [W]), there is an open cover $थ$ of $f(M)$ refining the collection of $\varepsilon / 4$-spheres, which is $\sigma$-discrete with respect to $\rho$ (or locally finite).

Thus $f^{-1}($ Q) is $\sigma$-discrete in $X$, with respect to $d(x, y)=\rho(f(x), f(y))$ (or locally finite). Now $\mathscr{U}$ consists of cozero-sets (because any open set in a metrizable space is cozero), and so does $f^{-1}(\mathscr{Q})$ (because $f^{-1}(\operatorname{coz} g)=$ $\operatorname{coz}(g \circ f))$. And $f$ oscillates $\leqslant \varepsilon / 2$ on each member of $f^{-1}(\mathcal{Q})$.

Evidently, $\cup f^{-1}(\mathcal{Q})=X \notin$ co $\mathscr{F}_{p}$. We claim that $f^{-1}(\mathscr{Q}) \subset$ co $\mathscr{F}_{p}$, i.e., that $p \in X-f^{-1}(U)$ for each $U \in \mathcal{Q}$ : for if not, and there is $U \in \mathcal{Q}$, and a neighborhood $G$ of $p$ with $G \cap\left(X-f^{-1}(U)\right)=\varnothing$, then $G \cap X \subset f^{-1}(U)$, and $\operatorname{osc}_{G} f \leqslant \varepsilon / 2$, a contradiction. Thus co $\mathscr{F}_{p}$ is not $\sigma$-discretely (or locally finitely) additive.

REMARK. The second half of the proof actually shows this. If $X$ is dense in $Y$, and if there is continuous $f: X \rightarrow M, M$ metrizable, which fails to extend to $p \in Y-X$, then there is a family $\left\{Z_{a} \mid a \in A\right\}$ of zero-sets (namely, the family $\left\{X-f^{-1}(U) \mid U \in \mathscr{Q}\right\}$ above) with $\left\{X-Z_{a} \mid a \in A\right\}$-discrete (or locally finite), with $\cap_{a} Z_{a}=\varnothing$ and $p \in \cap_{a} \bar{Z}_{a}$, the closures taken in $Y$.

3. COROLlaRY. $X$ is topologically complete iff each $z$-ultrafilter $\mathscr{F}$ for which coF is $\sigma$-discretely (or locally finitely) additive is of the form $\{Z \mid p \in \bar{Z}\}$ for some unique $p \in X$. 
Proof. $X \in \Re(\Re)$ iff $\delta X=X$.

The following useful proposition from [GJ] results from the association between $z$-ultrafilters and points of the epireflections $\beta X$ or $v X$.

4. Proposition. Let $X$ be dense in $Y$. The following are equivalent.

(a) Each continuous function from $X$ to $[0,1]$ (respectively, $R$ ) extends continuously over $Y$.

(b) $Y \subset \beta X$ (respectively, $Y \subset v X)$.

(c) If $\left\{Z_{a} \mid a \in A\right\}$ is a finite (respectively, countable) family of zero-sets of $X$ with $\cap_{a} Z_{a}=\varnothing$ then $\cap_{a} \bar{Z}_{a}=\varnothing($ the closures in $Y)$.

Analogously, we can derive easily from 2 the following.

5. Corollary. Let $X$ be dense in $Y$. The following are equivalent.

(a) Each continuous function from $X$ to a metrizable space extends continuously over $Y$.

(b) $Y \subset \delta X$.

(c) If $\left\{Z_{a} \mid a \in A\right\}$ is a family of zero-sets, with $\left\{X-Z_{a} \mid a \in A\right\} \sigma$-discrete (or locally finite), and if $\cap_{a} Z_{a}=\varnothing$, then $\cap_{a} \bar{Z}_{a}=\varnothing$ (the closures in $Y$ ).

Proof. (a) implies (b): Assuming (a), continuous functions from $X$ to metrizable spaces extend over $\delta Y$. By uniqueness of epireflections, $\delta Y=\delta X$ (essentially), so $Y \subset \delta X$ (essentially).

(b) implies (c): Assume (b), and let $\left\{Z_{a}\right\}$ be a family as in (c), with $\bigcap_{a} \bar{Z}_{a} \neq \varnothing$. Thus, for some $p \in \delta X, p \in \bigcap_{a} \bar{Z}_{a}$, and each $Z_{a} \in \mathscr{F}_{p}$. By $2, \mathscr{F}_{p}$ is $\sigma$-discretely or locally finitely additive, so $\bigcap_{a} Z_{a} \in \mathscr{F}_{p}$. Since $\mathscr{F}_{p}$ is a filter, $\cap_{a} Z_{a} \neq \varnothing$.

(c) implies (a): See the remark after 2 .

REMARKS. (1) A proof of 4 is easily constructed by analogy with the above proof of 5 .

(2) As noted in the introduction, the extension properties in 4(a) and 5(a) imply the stronger extension properties for maps into the epireflective hulls $\Re([0,1])=$ compact spaces, $\Re(R)=$ realcompact spaces, $\Re(\Re)=$ topologically complete spaces.

We conclude with a relatively simple proof, based on 2, of the KatětovShirota Theorem-or more exactly, of a version of the Gillman-Jerison version of the theorem. (See [K], [S], [GJ].)

Recall that the set $S$ has measurable power if there is a "nontrivial measure on $S$ ", i.e., a countably additive measure $\mu$, defined for all subsets of $S$, taking values 0 and 1 , with $\mu(\{p\})=0$ for each $p \in S$, and $\mu(S)=1$.

6. THEOREM. $\delta X=v X$ iff each discrete subset of $X$ has nonmeasurable power.

Proof. What we shall show is that $\sigma$-additivity of co $\mathscr{F}$ implies $(\sigma$-) discrete additivity for each $z$-ultrafilter iff the stated condition holds.

The "if" part is immediate from the following.

7. Lemma. Let $\mathscr{F}$ be a $z$-ultrafilter. If co $\mathscr{F}$ is $\sigma$-additive, then co $\mathscr{F}$ is "nonmea surably" discretely additive.

Proof. Let co $\mathscr{F}$ be $\sigma$-additive, let $\mathscr{D} \subset$ co $\mathscr{F}$ be discrete of nonmeasurable power. For $\mathscr{C} \subset \mathcal{D}$, define $\mu(\mathbb{Q})=1$ if $\cup \mathbb{Q} \notin \operatorname{co} \mathscr{F} ; \mu(\mathbb{Q})=0$ if $\cup \mathbb{Q} \in \operatorname{co} \mathscr{F}$. 
Note that for each $\mathscr{Q} \subset \mathscr{D}, \cup \mathbb{Q}$ is a cozero set because $\mathscr{Q}$ is discrete. For $D \in \mathscr{D}, \mu(\{D\})=0$ because $\mathscr{D} \subset$ co $\mathscr{F}$. We shall check that $\mu$ is a measure; thus $\mu$ will be identically $0, \mu(\mathscr{D})=0$ and $\cup \mathscr{D} \in$ co $\mathscr{F}$.

Let $\mathbb{Q}_{1}, \mathbb{Q}_{2}, \ldots$ be a sequence of disjoint subsets of $\mathscr{D}$. We shall show that $\mu\left(\cup_{n} \mathbb{Q}_{n}\right)=\Sigma_{n} \mu\left(\mathbb{Q}_{n}\right)$. Let $A_{n}=\cup\left\{D \in \mathbb{Q}_{n}\right\}, A=\cup_{n} A_{n}$. If $\mu\left(\cup_{n} \mathbb{Q}_{n}\right)=0$, then $A \in$ co $\mathscr{F}$.

For each $n, A_{n} \subset A$, hence $A_{n} \in$ co $\mathscr{F}$ and $\mu\left(Q_{n}\right)=0$. Thus $\mu\left(\cup_{n} \mathbb{Q}_{n}\right)=0$ $=\sum_{n} \mu\left(\mathbb{Q}_{n}\right)$. Now suppose $\mu\left(\cup_{n} \mathbb{Q}_{n}\right)=1$, i.e., $A \notin$ co $\mathscr{F}$. By $\sigma$-additivity, there is $n_{0}$ such that $A_{n_{0}} \notin$ co $\mathscr{F}$. Let $n \neq n_{0}$, then $\mathbb{Q}_{n} \cap \mathbb{Q}_{n_{0}}=\varnothing, A_{n} \cap A_{n_{0}}$ $=\varnothing$, and $\left(X-A_{n}\right) \cup\left(X-A_{n_{0}}\right)=X \in \mathscr{F}$. Since $\mathscr{F}_{F}$ is an ultrafilter, it is prime, and since $X-A_{n_{0}} \notin \mathscr{F}$, we have $X-A_{n} \in \mathscr{F}, A_{n} \in$ co $\mathscr{F}$, and $\mu\left(\mathbb{Q}_{n}\right)$ $=0$. So $1=\mu\left(\cup_{n} \mathbb{Q}_{n}\right)=\mu\left(\mathbb{Q}_{n_{0}}\right)=\sum_{n} \mu\left(\mathbb{Q}_{n}\right)$.

Conversely, let $X$ contain the discrete set $D$ of measurable power, and let $\mu$ be a nontrivial measure on $D$. Let $\mathscr{F}$ be the family of zero-sets $Z$ of $X$ with $\mu(Z \cap D)=1$. Evidently, $\mathscr{F}$ is a filter with co $\mathscr{F} \sigma$-additive.

We shall show that $\mathscr{F}$ is maximal and not discretely additive. For use in both parts, choose $d$ and $\varepsilon$ with $d(p, q) \geqslant \varepsilon$ for $p \neq q$ in $D$, and for $p \in D$, set $C_{p}=\{x \mid d(p, x)<\varepsilon / 4\}$. Note that $C_{p}$ is a cozero-set, and $\left\{C_{p} \mid p \in \mathscr{D}\right\}$ is discrete.

$\mathscr{F}$ is maximal: Let $Z_{0}$ be a zero-set with $Z_{0} \cap Z \neq \varnothing$ for each $Z \in \mathscr{F}$. For each $p \in D-Z_{0}$, choose a zero-set $Z_{p}$ with $p \in Z_{p}, Z_{p} \cap Z_{0}=\varnothing$, and $Z_{p} \subset C_{p}$. Let $C_{p}=\operatorname{coz} f_{p}$ and $Z_{p}=Z g_{p}$. Then $\left\{\operatorname{coz}\left(f_{p} g_{p}\right) \mid p \in D-Z_{0}\right\}$ is discrete, and with $f=\sum\left\{f_{p} g_{p} \mid p \in D-Z_{0}\right\}$, we have $Z f=\cup\left\{Z_{p} \mid p \in D\right.$ $\left.-Z_{0}\right\}$. Now $Z_{0} \cap Z f=\varnothing$, so that $Z f \in \mathscr{F}$. Since $D=(Z f \cap D) \cup\left(Z_{0}\right.$ $\cap D)$, it follows that $\mu\left(Z_{0} \cap D\right)=1$, and $Z_{0} \in \mathscr{F}$.

$\mathscr{F}$ is not discretely additive: Let $\mathscr{D}=\left\{C_{p} \mid p \in D\right\}$. Evidently, $\mathscr{D} \subset$ co $\mathscr{F}$, while $\mu((X-\cup \mathscr{D}) \cap D)=\mu(\varnothing)=0$, so that $\cup \mathscr{D} \notin$ co $\mathscr{F}_{p}$.

RemarKs. (1) It is easy to show that each discrete subset of $X$ has nonmeasurable power iff each locally finite subset of $X$ has nonmeasurable power. This yields another version of 6 .

(2) The proof of 6 given above (including the proof of 2) resembles to some degree the proof of 15.21 of [GJ]. 7 generalizes 12.3 of [GJ].

(3) All known proofs of theorems close to 6 use the Stone Theorem on $\sigma$ discrete refinement. Our use of it is confined to 2, and our proof of 6 proper consists of fairly simple set-theoretic computations.

(4) $[\mathrm{DW}]$ and 2.4 of [T] use locally finite partitions of unity to characterize topological completeness; the ideas are somewhat similar to those of this paper.

(5) The referee points out that the space we are labelling $\delta X$ (after its inventor Dieudonné [D]) has been labelled $\theta X$ in [B] and $\mu X$ in [M].

(6) The referee points out that Buchwalter [B] has also and earlier obtained an identification of the points of $\delta X$ among those of $v X$ : As is well known, the points $p$ of $v X$ (i.e., the $z$-ultrafilters $\mathscr{F}$ with co $\mathscr{F} \sigma$-additive) correspond one-toone with the unitary ring homomorphism $h: C(X) \rightarrow R$. In $\$ 4$ of [B], Buchwalter shows that the following conditions on $h$ are equivalent: (a) $h \in \delta X$. (b) $h \mid E$ is continuous for every equicontinuous $E \subset C(X), E$ having the topology of simple convergence on $X$. (c) If $\left\{E_{n}\right\}$ is a sequence of equicontinuous subsets of $C(X)$, then there is $x \in X$ such that $h(f)=f(x)$ for each $f \in \cup_{n} E_{n}$. 
As here 2 implies 6, the above implies a version of the Katětov-Shirota Theorem ([B, p. 55]).

Buchwalter's Theorem and our 2 establish that a homomorphism $h$ satisfies (b) above iff the associated $z$-ultrafilter $\mathscr{F}=\{Z(f) \mid f \in \operatorname{ker} h\}$ has co $\mathscr{F} \sigma$ discretely additive.

(7) As is well known (e.g., [D]), $\delta X$ can be viewed as the topological space underlying the completion of $X$ equipped with its finest compatible uniformity. We have chosen to avoid connections with uniformities here, but a later paper $[\mathbf{C H}]$ will treat this thoroughly.

\section{BIBLIOGRAPHY}

[B] H. Buchwalter, Topologies et compactologies, Publ. D. Math. (Lyon). 6 (1969), 1-74.

[CH] Howard Curzer and Anthony W. Hager, $z$-ultrafilters in uniform spaces (in preparation).

[DW] G. DeMarco and R. G. Wilson, Realcompactness and partitions of unity, Proc. Amer. Math. Soc. 30 (1971), 189-194. MR 43 \#6874.

[D] J. Dieudonné, Sur les espaces uniformes complets, Ann. Sci. École Norm. Sup. 56 (1939), 277-291. MR 1, 220.

[F] S. P. Franklin, On epi-reflective hulls, General Topology and Appl. 1 (1971), no. 1, 29-31. MR 44 \#3287.

[GJ] L. Gillman and M. Jerison, Rings of continuous functions, University Ser. in Higher Math., Van Nostrand, Princeton, N.J., 1960. MR 22 \#6994.

[H] Anthony W. Hager, Perfect maps and epi-reflective hulls, Canad. J. Math. 27 (1975), 11-24.

[HS] H. Herrlich and J. van der Slot, Properties which are closely related to compactness, Nederl. Akad. Wetensch. Proc. Ser. A 70 = Indag. Math. 29 (1967), 524-529. MR 36 \# 5898.

[K] M. Katětov, Measures in fully normal spaces, Fund. Math. 38 (1951), 73-84. MR 14, 27.

[M] K. Morita, Topological completions and M-spaces, Sci. Rep. Tokyo Kyoiku Daigaku Sect. A. 10 (1970), 271-288. MR 42 \#6785.

[S] T. Shirota, A class of topological spaces, Osaka Math. J. 4 (1952), 23-40. MR 14, 395.

[St] A. H. Stone, Paracompactness and product spaces, Bull. Amer. Math. Soc. 54 (1948), 977-982. MR 10, 204.

[T] H. Tolmano, Some properties of the Stone-Čech compactification, J. Math. Soc. Japan 12 (1960), 104-117. MR 26 \#6929.

[W] S. Willard, General topology, Addison-Wesley, Reading, Mass., 1970. MR 41 \#173.

Czechoslovakian Academy of Sciences, Prague, Czechoslovakia

Department of Mathematics, Wesleyan University, Middletown, Connecticut 06457 